to what he saw. The best method of retinoscopy was by means of slit-lamp and a non-silvered mirror, i.e., a plate of glass with two plain faces. If that plan were followed it would not be necessary to use a cycloplegic. Mr. W. H. Brailey thought that the manifest hypermetropia should be taken first, and that when there was a large discrepancy between it and the final result a cycloplegic was indicated. He considered that mydriatics were too much used in myopia; they were seldom necessary. $\mathrm{Mr}$. Ernest Clarke had tested one hundred consecutive cases without a cycloplegic, and had then put them under atropin or homatropin and found that in seventy-six cases the results were different. $\mathrm{Mr}$. N. Bishop Harman was convinced of the value of an oily preparation of homatropin and cocain as a cycloplegic in suitable cases. $\mathrm{Mr}$. Claud Worth practically never used homatropin for purposes of refraction, but for small children employed atropin for three days. Mr. A. C. Brinton, who practises in Johannesburg, spoke of the asthenopia often found at that altitude in patients with a small amount of ametropia. Mr. A. Hugh Thompson did not believe that the refraction between the macula and the disc was the same, at any rate, in myopia. Mr. Traquair believed all Edinburgh ophthalmologists thought that the use of a cycloplegic had to be determined in each individual case. The Edinburgh practice was to use a cycloplegic for children, but not for adults. $\mathrm{Mr}$. Stack said one reason for using a cycloplegic was when hyperphoria was present. Before ordering a prism it was advisable to ascertain that hyperphoria was still present under the cycloplegic.

It is gratifying to note the continued prosperity of the Society, of which there are 482 subscribing and five honorary members. One criticism on the balance-sheet may be permitted. The Nettleship Prize Fund, which is vested in four trustees appointed for the purpose by the Society, is not provided, as it should be, with a separate balance-sheet apart from the accounts of the Society.

S. S.

\title{
CORRESPONDENCE
}

\section{TEST TYPES}

\section{To the Editor of The British Journal of Ophthalmology}

Sir,--Many readers of the journal will have appreciated the patient work of Drs. Hartridge and Owen, as shown in the paper on "Test Types" which appeared in the December issue..

Having at one time given a great deal of time and thought to the subject, I was particularly interested in the authors' experi- 
ments, all the more so because the results seemed on first sight to favour a different set of letters from those I had chosen for my own test types. Upon examining the results more carefully, however, I find that, far from justifying the authors' conclusions, they, in the main, support my own choice of letters.

The table given on page 545 shows the results in tabular form. The letters $\mathrm{S}$ and $\mathrm{R}$ are obviously useless, and need not detain us. Taking the remainder of the letters examined, we will, for a moment, omit the tests beyond the distance of 8.50 metres, because then Dr. Hartridge made so many mistakes that he was clearly at a loss most of the time. And so, confining ourselves to the first three distances, viz., 5 metres, 7.50 metres, and 8.50 metres, we find that Dr. Hartridge made 53 mistakes, and Dr. Owen made 18. The latter's visual acuity is, therefore, almost precisely three times better than that of the former. It is so good, indeed, that we are most anxious to learn the results at the 10 metre distance. Here we find his best letters (taking those in which he made no mistakes or.only one) are $T, \mathrm{Z}, \mathrm{P}, \mathrm{X}, H, N, E, \mathrm{~J}, A, L . \quad$ I have used italics for those which figure in my test types.

Regarding $\mathrm{Z}$ and $\mathrm{X}$, the authors quote me as saying that I " left [them] out because they are not so familiar as the others." What I did say in the paper referred to (Trans. of the Ophthal. Soc., Vol. XXXIX, 1919, p. 242), I will, with your permission, quote in full :- " Long experience in examining school children taught me to leave out the letters $X$ and $Z$. They are not homely letters. Children do not become familiar with them until they are well on in the curriculum. Even adults stumble over them. And so they are best left out." I am sure that every oculist who has much to do with school children will agree that my opinion is abundantly justified. The authors wish to retain them because they think that these two letters are valuable for testing cases of astigmatism, but who, in this the twentieth century, uses them for the purpose of testing for astigmatism? I submit that whatever their advantages may be, they are far exceeded by their disadvantages. They cause an immense loss of time.

There remain the letters $J$ and $P$. J, unless the tail is exaggerated, has the same defects as I, and the authors have rightly omitted the letter. P has come out of the test much better than I thought it would, but Dr. Owen made a mistake with it at 8.50 metres.

The authors say that $\mathrm{S}, \mathrm{R}, \mathrm{B}$, and $\mathrm{O}$ are more difficult to read at great distances because they approximate to the grating in form. It is strange to allege this against $\mathrm{O}$ and to omit $\mathrm{P}$.

Omitting $\mathrm{X}, \mathrm{Z}$, and $\mathrm{J}$ for the reasons given, and $\mathrm{P}$, undoubtedly $T, H, N, E, A, L$, are Dr. Owen's best letters, and if we add to these $\mathrm{O}$ and $\mathrm{C}$ we have the same set as I have used for my test types.

The authors exclude $\mathrm{O}$ and $\mathrm{C}$. In my opinion, Dr. Owen would 
have done as well with these two letters as with the others if they had differed from each other sufficiently to emphasize their difference rather than their similarity. In my own test types, the letter $\mathrm{C}$ is never mistaken for any other letter. Even so, Dr. Owen made no mistake with $\mathrm{C}$ at 8.50 metres, and only one mistake with $O$. They are clearly good letters. Moreover, they are letters with which children are most familiar. They cannot possibly be left out of any useful set of test types.

Drs. Hartridge and Owen have chosen what they call " letters of medium difficulty." The reason for the choice does not appear, but the more simple a test the greater its accuracy and value as an instrument of precision, and the more simple the letters the better our test types will be for the purpose intended.

As far as the shape of the letters is concerned, there seems to be little or nothing to choose between Snellen's $5 \times 5$ and Green's $4 \times 5$ on the score of visibility, with the possible exception of $\mathrm{O}$, which would be more easily read when round than when oval.

There are many other points of interest in the paper, but I have already trespassed sufficiently upon your valuable space.

SHEFFIELD. Yours truly,

Percival J. Hai.

\section{GERMAN OPHTHALMOLOGICAL LITERATURE}

To the Editor of The British Journal of Ophthalmology

SIR,-In view of the letter in your last issue from Julius Springer, the following facts may be of interest. Towards the end of last year, I had occasion to order from Berlin the numbers of the Jahresbericht which have been published since the war broke out, viz. : Vols. XLIV and XIVII. The price paid was as stated in the publisher's letter, 40s. for Vol. XLIV, 32s. for Vol. XLVII.

Last week I received by post a second copy of Vol. XLVII, with a bill enclosed for $£ 1$ 1s. 6 d.

Your annotation, Sir, seems to have had the effect of reducing the price of this work by a considerable amount. I can offer no suggestion as to why I have received a second copy which I did not ask for. It may be a subtle form of humour on the publisher's part; he may be under the impression that I wrote the annotation in question; allow me to state that such is not the case, and to inform him through the medium of your pages that he may have his spare copy by instructing his representative in this country to call at my address on any morning between the hours of 9.30 and 11.30 a.m.

46, Wimpole Street, W.1.

Yours faithfully,

R. R. JAMEs. 